\title{
Verrucous Papilloma
}

National Cancer Institute

\section{Source}

National Cancer Institute. Verrucous Papilloma. NCI Thesaurus. Code C4101.

A benign epithelial neoplasm characterized by a papillary growth pattern, lack of significant cytologic atypia, and a wart-like appearance. 